\title{
Política de ciência, tecnologia e inovação no Brasil após 2000: contrapondo reflexões e indicadores
}

\author{
Christian Luiz da Silva*
}

Nádia Solange Schmidt Bassi**

Fabiana Ieis ${ }^{* * *}$

\begin{abstract}
RESUMO - O objetivo do artigo é identificar a trajetória das políticas brasileiras de Ciência, Tecnologia e Inovação (C\&TI) a partir da década de 2000. Foram selecionados estudos recentes sobre o tema para avaliar o principal objeto de discussão, os quais foram comparados com as principais políticas desenvolvidas e os indicadores de ciência, tecnologia e inovação. A literatura sobre o assunto aponta que o país tem buscado, por meio da agenda de política de C\&TI, a consolidação da base científica e tecnológica, mas que não tem considerado sua situação de país em desenvolvimento, sua trajetória histórica de dependência dos países centrais e sua realidade de desigualdade social e econômica, que se reflete no desenvolvimento científico e tecnológico. Essa dificuldade retrata-se na evolução dos indicadores que demonstram um baixo dinamismo de inovação e de inserção de novos doutores na indústria brasileira, a despeito das políticas de incentivo do período.
\end{abstract}

Palavras-chave: Política científica e tecnológica. Indicadores de inovação. Inovação tecnológica. Brasil.

\section{INTRODUÇÃO}

A questão tecnológica e da inovação está relacionada ao processo de desenvolvimento dos países e do sistema capitalista, como fundamenta Schumpeter (1997), dentre outros. A despeito da controvérsia entre o determinismo, a relação minimizadora dos riscos e incertezas a partir das políticas de Ciência e Tecnologia (C\&T), esta trata de uma intervenção do Estado para criar um ambiente favorável ao desenvolvimento tecnológico e à inovação (LATOUR, 1983; ANDRADE, 2004). A intervenção pode ocorrer de diversas maneiras e as suas compreensão e construção histórica permitem avaliar o papel do Estado e governos neste processo, assim como sua efetividade. O presente artigo pretende identificar a trajetória das políticas

\footnotetext{
* Doutor em Engenharia de Produção pela Universidade Federal de Santa Catarina. É professor do Programa de Doutorado em Tecnologia e coordenador do programa de Pós-Graduação em Planejamento e Governança Pública da Universidade Tecnológica Federal do Paraná. Endereço eletrônico: christiansilva@utfpr.edu.br.

** Mestranda em Tecnologia e Sociedade pela Universidade Tecnológica Federal do Paraná. É pesquisadora da Embrapa. Endereço eletrônico: sbnadia@hotmail.com.

**** Mestranda em Tecnologia pela Universidade Tecnológica Federal do Paraná. Endereço eletrônico: fabi_ieis@, hotmail.com.
} 
brasileiras de Ciência, Tecnologia e Inovação (C\&TI) a partir da década de 2000. Para isso, analisam-se alguns estudos realizados sobre as políticas de C\&TI, os esforços despendidos no Brasil em $\mathrm{P} \& \mathrm{D}$ e os indicadores relacionados às políticas de ciência, tecnologia e inovação, a partir de dados elaborados pelo Ministério da Ciência e Tecnologia (MCT).

\section{AS VISÕES DA LITERATURA SOBRE A PNCT E OS INDICADORES DE INO- VAÇÃO NO BRASIL}

A evolução das ações de Política de Ciência e Tecnologia no Brasil foi contraposta com a discussão acadêmica. O desenvolvimento da ciência e da tecnologia tem se mostrado como condição necessária, ainda que não suficiente, para o desenvolvimento do país, o que se retrata em um extenso volume de publicações visando discutir as políticas de C\&TI no Brasil.

Um dos pontos mais recorrentes na literatura sobre as políticas de C\&TI é a condição de país periférico no qual o Brasil se inclui. Para Motoyama (2004), o legado de uma cultura estagnada e descrente do pensamento científico e tecnológico persegue a trajetória da C\&TI com o peso da dependência percebida no decorrer da história do país.

Outra característica apontada na literatura é o da centralização das ações políticas. No Brasil, mais de dois terços do sistema de ciência e tecnologia está concentrada na região Sudeste (SILVA, 2000), o que indica mais uma bifurcação dos caminhos da política de C\&TI para nossa situação de dependência histórica de desenvolvimento.

Entretanto, como afirma Corrêa (2003), as políticas nessa área aparentam acompanhar o modelo internacional em busca de inovações que possam ser incorporadas nos mercados internacionais. Apoiar-se em exemplos de países considerados do centro pode condenar formas alternativas de produção técnica e científica que poderiam ser mais interessantes e eficazes para o desenvolvimento dos países periféricos, como é o caso do Brasil (DAGNINO; DIAS, 2007).

Portanto, para uma real avaliação da consolidação da base científica no Brasil, é preciso não somente levar em conta as leis estabelecidas, mas também no que elas estão apoiadas e nos resultados que estão apresentando. Para ajudar nessa avaliação, um olhar sobre alguns pontos da literatura produzida acerca desse assunto é apresentado resumidamente na Tabela 1.

Esse contexto de esforço da Política Nacional de Ciência e Tecnologia no Brasil inclui preceitos importantes, mas sua efetividade é condicionada por uma série de fatores e condições institucionais que demandam uma análise dos indicadores de inovação no país. 
TABELA 1 - TRAJETÓRIA E PERSPECTIVA DE ESTUDOS SELECIONADOS SOBRE POLÍTICA DE CIÊNCIA, TECNOLOGIA E INOVAÇÃO NO BRASIL

\begin{tabular}{|c|c|c|}
\hline Estudo & Trajetória & Perspectivas \\
\hline $\begin{array}{l}\text { Descentralização em } \\
\text { política de ciência e } \\
\text { tecnologia } \\
(\text { SILVA, 2000) }\end{array}$ & $\begin{array}{l}\text { As ações das políticas de C\&T no Brasil } \\
\text { estão concentradas na região sudeste, as } \\
\text { regiões menos favorecidas ficam privadas de } \\
\text { desenvolver pesquisa aplicada ao seu desen- } \\
\text { volvimento e de incorporar conhecimentos } \\
\text { gerados em outros centros. }\end{array}$ & $\begin{array}{l}\text { O Brasil já conta com instrumentos que lhe } \\
\text { permitem descentralizar a política de ciência } \\
\text { e tecnologia, atendendo às condições e } \\
\text { oportunidades em cada região. A política de } \\
\text { C\&TI deve incluir em suas ações propostas } \\
\text { para essa descentralização. }\end{array}$ \\
\hline
\end{tabular}

A política de C\&T A tentativa de alcançar os países centrais em É preciso discutir escolhas que possibilitem brasileira: três alter- termos de desenvolvimento científico e tec- orientar a política pública de C\&T com nativas de explica- nológico representa um equívoco, e apesar mecanismos de controle mais democráção e orientação desse fato ser debatido desde os anos 70 , (DAGNINO; DIAS, ainda continua instaurado na percepção da 2007) comunidade de pesquisa latino-americana.

Os fundos setoriais As diversas tentativas empreendidas pelo e a política nacional Estado brasileiro para minimizar as limitade ciência, tecno- ções que comprometiam uma maior amlogia e inovação plitude produziram uma série de gargalos, (VALLE et al., 2002) ocasionando restrições ao desenvolvimento econômico e social, aos esforços de universidades e institutos de pesquisa e à competitividade de empresas e organizações, uma vez que quase todos os projetos apoiados envolveram o setor privado.

Política científica e tecnológica e a dinâmica inovativa no Brasil (BAGATTOLLI, 2008)

As características da atual política científica explícita são marcadas por três tendências que se entrelaçam. São elas: i) o aumento dos recursos públicos para atividades de ticos e com valores coerentes a um estilo de desenvolvimento sustentável no longo prazo, de forma que não condene as formas alternativas de produção de conhecimento técnico e científico que poderiam representar estratégias mais interessantes para o desenvolvimento dos países periféricos, como é o caso do Brasil.

Há a necessidade de criar projetos que integrem a pesquisa pública e o setor privado, na condição de agente financiador e executor de atividades de pesquisa.

O que se verifica é que, ao invés desses problemas se amenizarem ou se resolverem, eles se reproduzem, dado que a resolução dos obstáculos estruturais decorrentes da nossa C\&TI; ii) ênfase aos projetos cooperativos e; condição periférica diz respeito à outras iii) de forma relativamente mais importante, políticas públicas que não a de C\&TI. aumento dos mecanismos de fomento à inovação empresarial.

Demandas empresa- Para as empresas, a inovação aparece em riais em políticas de segundo plano e a tecnologia não é consideciência, tecnologia e rada como urgente.

inovação no Brasil a Existem algumas ilhas de excelência em partir dos anos 1990 termos de empresas, que reconhecem a (HIRATA, 2006) importância da inovação, porém essa não é a realidade da maioria das empresas que não têm tradição em inovação.

O Brasil na era do conhecimento: políticas de ciência e tecnologia e desenvolvimento sustentado (CORRÊA, 2003)

A partir de 1990 as políticas de C\&TI são voltadas, em sua maioria, para as agendas de competitividade - obedecendo a condição periférica do país, essas políticas trazem benefícios para poucas empresas. São políticas verticalizadas, ausentes de planejamento global.

FONTE: Adaptado de SILVA (2000), DAGNINO e DIAS (2007), VALLE et al. (2002), BAGATTOLLI (2008),
É importante a participação do governo, da academia e das empresas no processo de inovação, que ainda é caracterizado como rudimentar (diferente de outros países, desenvolvido com método sistêmico de inovação), produzindo barreiras à interação entre as instituições que promovam ou orientem os processos de inovação. Para isso, a inovação deve ser considerada política de Estado, não de um governo ou outro.

Políticas baseadas em um modelo internacional, que se mostram inadequadas dada nossa condição periférica. É necessário estabelecer uma agenda científica que possa ser debatida amplamente pela coletividade científica, Estado e a sociedade civil.

HIRATA (2006) e CORRÊA (2003). 


\subsection{INDICADORES DE INOVAÇÃO}

Os indicadores de inovação se apresentam como outro ponto de avaliação das políticas empreendidas. Para um melhor entendimento do esforço despendido pelo país para melhorar seu índice de desenvolvimento tecnológico e inovação, torna-se imprescindível analisar alguns dos dados diretamente relacionados à estes fatores.

\subsection{O DISPÊNDIO NACIONAL EM P\&D}

O Brasil tem investido de forma inconstante os recursos públicos no desenvolvimento de ciência e tecnologia. Em 2001, foi aplicado 1,30\% do PIB em C\&T. Seis anos depois o percentual chegou a apenas a 1,46\%, enquanto que a Venezuela despendeu 2,69\% do seu PIB (Gráfico 1). Segundo o MCT (2002), em 2012 este índice deverá chegar a 2\%, equiparando-se aos países desenvolvidos.

\section{GRÁFICO 1 - APLICAÇÃO DO PIB EM CIÊNCIA E TECNOLOGIA}

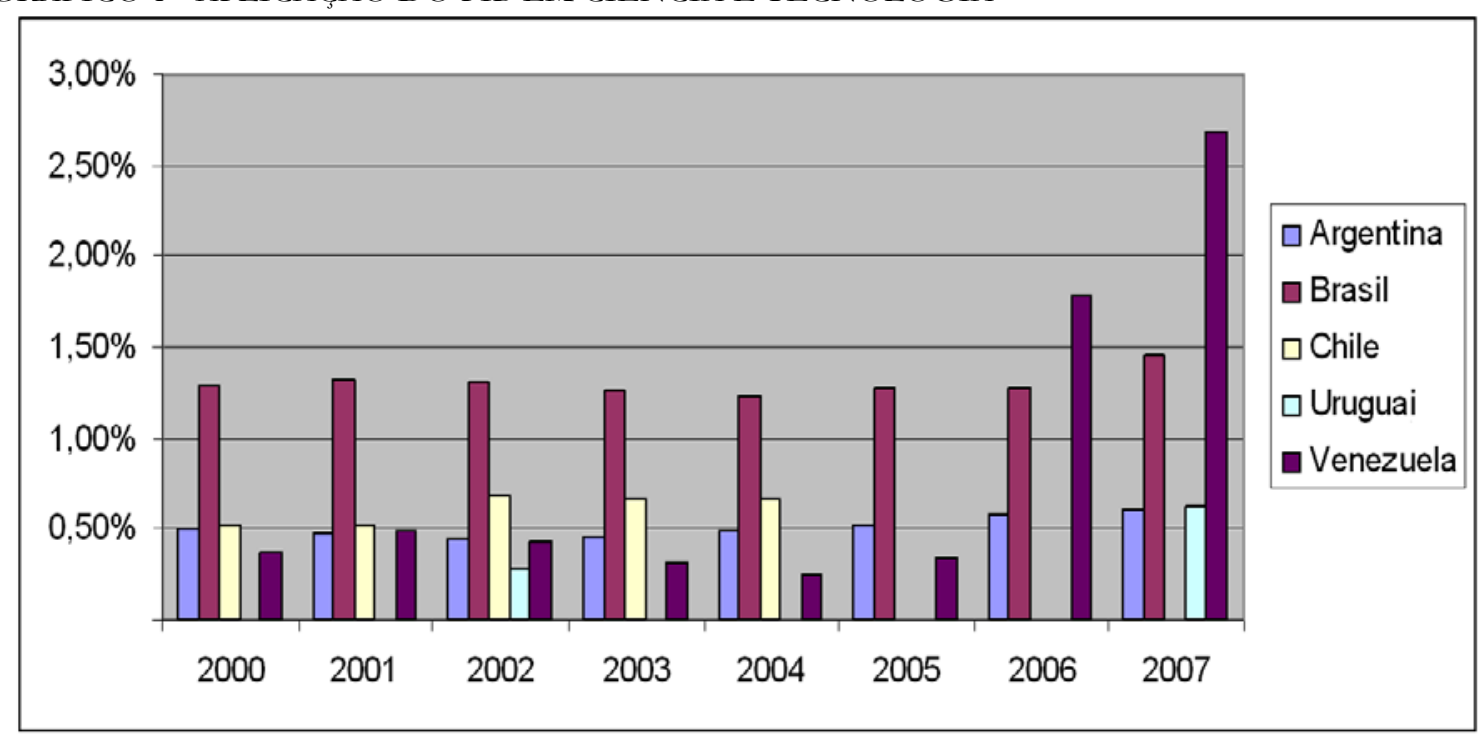

FONTE: MCT, 2011.

Os dispêndios públicos e privados apontam para um grande desafio para as políticas para C\&TI no Brasil: a criação de um ambiente que estimule e viabilize o aumento no dispêndio empresarial em P\&D. Isso é bem mais complexo do que o aumento do dispêndio público, pois envolve obter um aumento substancial no investimento privado por meio de políticas governamentais (CRUZ, 2010).

\subsection{AS PATENTES NO BRASIL}

Apesar da criação de diversas leis para regularizar as obrigações e direitos sobre a propriedade intelectual (Lei de Patentes - 9.279/96; Lei de Proteção de Cultivares - 9.456/97; 
Lei de Informática - 11.077/04) e de leis de incentivo à inovação tecnológica (Lei de Inovação - 10.973/05; Lei do Bem - 11.196/05 e a Lei Rouanet da Pesquisa - 11.487/07), o Brasil ainda tem um nível bastante baixo de patentes, principalmente de residentes (Gráfico 2). Conforme Sennes (2009), o país figura na $28^{a}$ posição no ranking mundial de patentes, com apenas 121 patentes obtidas em 2006 no United States Patent and Trademark Office (USPTO).

GRÁFICO 2 - PEDIDOS DE PATENTE DEPOSITADOS NO INPI, POR RESIDENTES E NÃO RESIDENTES (1990-2010)

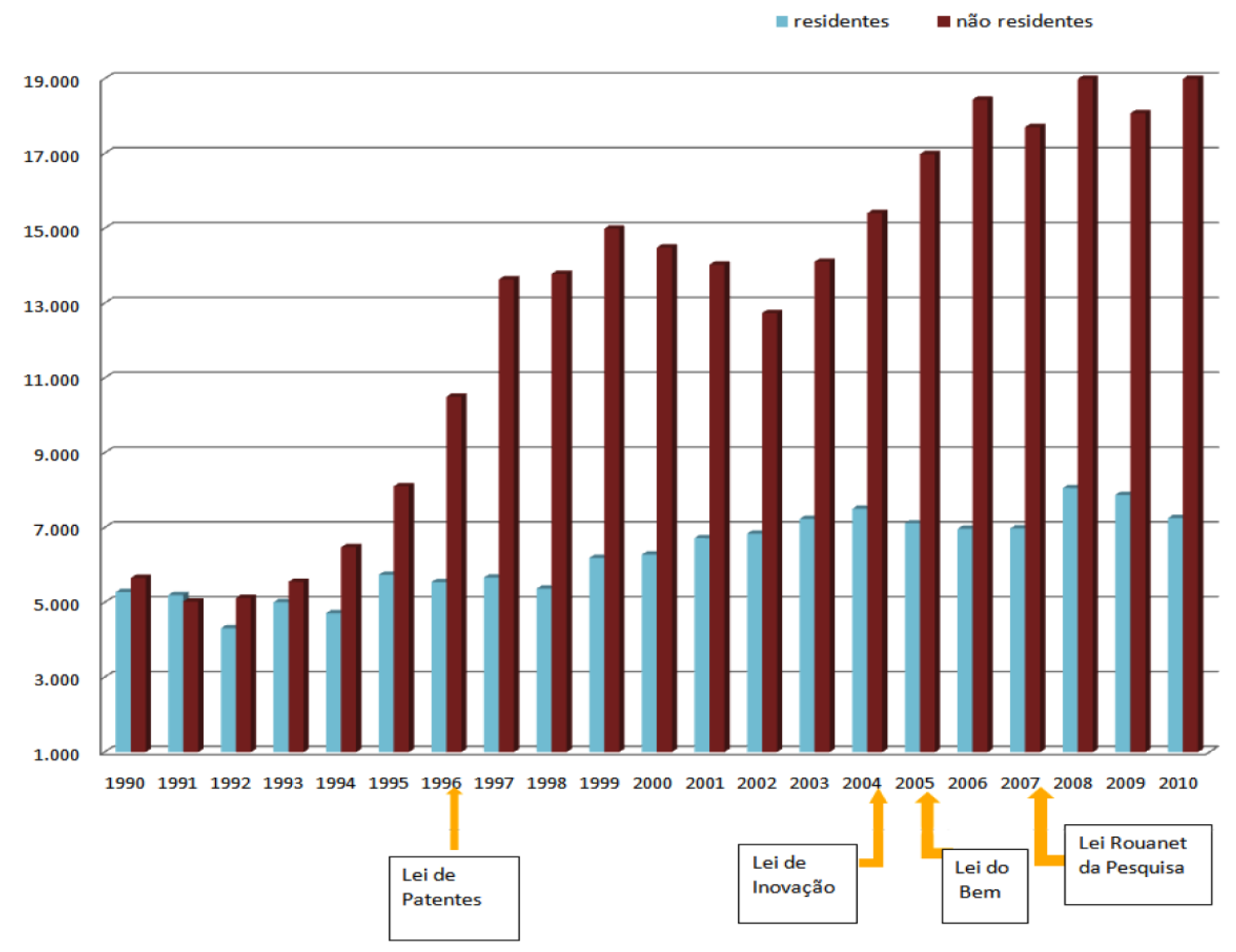

FONTE: MCT, 2011.

Os dados demonstram que nos últimos catorze anos o número de pedidos de patente por residentes teve um aumento de apenas 31\%, passando de 5.540 pedidos em 1996 para 7.256 em 2010, enquanto que o depósito por não residentes no Brasil teve um incremento ao redor de 98\%. Esta tendência manteve-se após a implantação da Lei de Inovação (2004) e da Lei do Bem (2005), o que leva a concluir que a criação destas leis não incentivou substancialmente o número de pedidos de patentes por brasileiros, como era esperado. Para explicar as razões dos números apresentados, há necessidade de se estudar de forma mais aprofundada estas leis, o que não é objetivo do presente artigo. 


\subsection{A CONTRATAÇÃO DE PESQUISADORES POR EMPRESAS PRIVADAS}

A Lei do Bem, que prevê incentivos fiscais à inovação tecnológica ligados a gastos com P\&D, como a contratação de pesquisadores para atividades de PD\&I por empresas privadas, também não apresentou resultados substanciais (Gráfico 3). Este fato pode ser constatado ao analisarmos o percentual de aumento de apenas $5 \%$ de pesquisadores atuantes no setor empresarial entre 2005 - ano da implantação da Lei do Bem - e o ano de 2007. Observa-se ainda que $71,31 \%$ dos pesquisadores na área de $\mathrm{P} \& \mathrm{D}$ encontram-se em universidades e somente $26,7 \%$ dos mesmos encontram-se no setor empresarial.

GRÁFICO 3 - PESQUISADORES ENVOLVIDOS EM PESQUISA E DESENVOLVIMENTO (P\&D), POR SETOR INSTITUCIONAL NO BRASIL, 2000-2008

moverno Ensino superior $=$ Empresarial $=$ Privado sem fins lucrativos

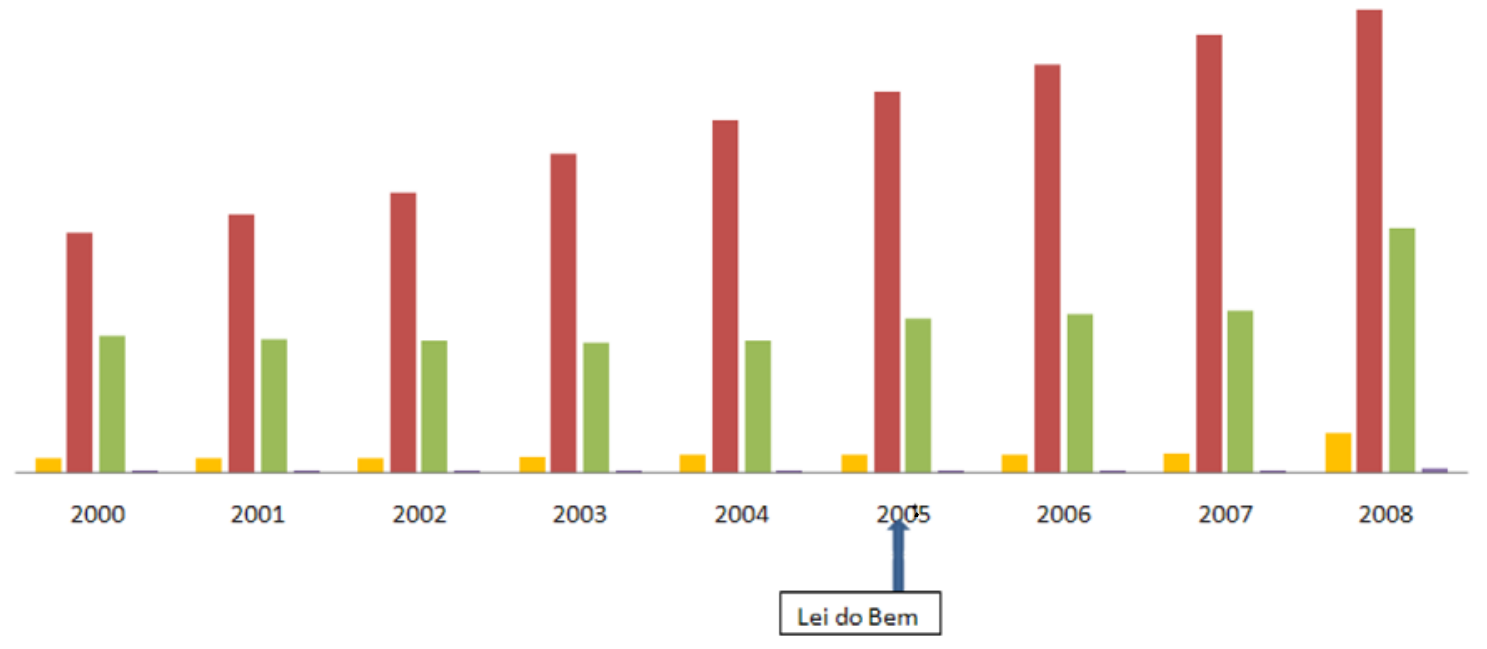

FONTE: MCT, 2011.

Os resultados apontados nos Gráficos 2 e 3 sugerem que a criação das leis de incentivo à inovação não resultaram em um aumento substancial dos índices ligados à questão da inovação tecnológica no Brasil.

\section{CONCLUSÃO}

As políticas de C\&T, isoladamente, não darão conta de mudar o ambiente institucional e integrar regiões e estratégias vinculadas de Governo e empresas. Denota-se que sua institucionalização ocorre por uma via burocratizada e complexa, que muitas vezes torna-a impraticável, tanto no que concerne a sua compreensão em termos de legislação quanto às possibilidades de sua utilização pelos meios público e privado. É importante ainda ressaltar que para a promoção da inovação no país é necessário o desenvolvimento de políticas públicas de longo prazo e reali- 
zadas em parceria com o setor privado, de modo a possibilitar o desenvolvimento tecnológico.

A revisão na política de C\&TI demonstra a existência de um ambiente institucional que se modificou no curto prazo, dificultando as decisões de longo prazo que dependem de uma ação transversal e intersetorial, por seu contexto histórico e institucional. Esta mudança exige uma visão de Estado (longo prazo) em detrimento ao planejamento tão somente de Governo (curto e médio prazos). A política de Governo deve estar inserida na política de Estado, permeando um ambiente institucional favorável de longo prazo, apoiado em ações de promoção de educação e cultura de inovação, estimulando a maior interação entre estratégias do Governo e das empresas. Cabe, com isso, compreender que muitos pontos discutidos na literatura recente são coerentes com os propósitos da polítca de C\&TI, mas que a prática desta política depende da incorporação destas discussões em estratégias de longo prazo para mudança da condição nacional em termos de inovação e desenvolvimento tecnológico.

Contudo, há de se enfatizar que refletir sobre as políticas de CT\&I no contexto brasileiro significa tratar de uma preocupação relativamente recente. Percebe-se que as políticas implantadas ainda não se consolidaram de forma efetiva no contexto social e num planejamento de médio e longo prazos.

\section{REFERÊNCIAS}

ANDRADE, T. de. Inovação tecnológica e meio ambiente: a construção de novos enfoques. Ambiente \& Sociedade. Campinas, v. 7, n. 1, p. 89-106, jan./jun. 2004.

BAGATTOLLI, C. Política cientifica e tecnológica e dinâmica inovativa no Brasil. Dissertação (Mestrado) - Instituto de Geociências, Universidade Estadual de Campinas, Campinas, 2008.

BRASIL. Ministério do Desenvolvimento, Política e Comércio Exterior - MDIC. PITCE 2003. Disponível em: < http://www2.desenvolvimento.gov.br/sitio/ascom/ascom/polindteccomexterior.php>. Acesso em: 10/3/2011.

BRASIL. Ministério da Ciência e Tecnologia - MCT. Indicadores. Disponível em: <http:// www.mct.gov.br/index.php/content/view/740.html?execview >. Acesso em: 12/3/2011.

CORRÊA, M. B. O Brasil na era do conhecimento: políticas de ciência e tecnologia e desenvolvimento sustentado. Tese (Doutorado) - Instituto de Filosofia e Ciências Humanas - Universidade Federal do Rio Grande do Sul, Porto Alegre, 2003.

CRUZ, H. de B. Ciência, tecnologia e inovação no Brasil: desafios para o período 2011 a 2015. Interesse Nacional, São Paulo, n. 9, abr./jun. 2010. Disponível em: $<$ http://www.ifi.unicamp. br/.../CTI-desafios-InteresseNacional-07082010-FINAL.pdf $>$.

DAGNINO, R. P.; DIAS, R. A política de C\&T brasileira: três alternativas de explicação e orientação. Revista Brasileira de Inovação, Rio de Janeiro, v. 6, n. 2, p. 373-403, 2007.

HIRATA, N. Demandas empresariais em políticas de ciência tecnologia e inovação no Brasil a partir dos anos 1990. Tese (Doutorado) - Faculdade de Filosofia Letras e Ciências 
Humanas, Universidade de São Paulo, São Paulo, 2006.

LATOUR, B. Give me a laboratory and I will raise the world. In: KNORR-CETINA, K.; MULKAY, M. (Ed.). Science observed: perspectives on the social study of science. Londres: Sage, 1983, p. 141-170.

MOTOYAMA, S. (Org.). Prelúdio para uma história - ciência e tecnologia no Brasil. São Paulo: Edusp/FAPESP, 2004.

SCHUMPETER, J. A. Teoria do desenvolvimento econômico. São Paulo: Nova Cultural, 1997.

SILVA, A. C. da. Descentralização em política de ciência e tecnologia. Estudos Avançados, São Paulo, v. 14, n. 39, p. 61-73, 2000. Disponível em: <http://www.scielo.br/scielo.php?pid=S0103$40142000000200007 \&$ script $=$ sci_arttext $>$. Acesso em 13/3/2011.

VALLE, M. G. do; BONACELLI, M. B. M.; SALLES FILHO, S. L. M. Os fundos setoriais e a política nacional de ciência, tecnologia e inovação. In: SIMPÓSIO DE GESTÃO DA INOVAÇÃO TECNOLÓGICA, 22, 2002, Salvador. Anais... Disponível em: < http:/ /www.ige.unicamp.br/geopi/documentos/22809819.pdf>. 